\title{
New numerical method based on Generalized Bessel function to solve nonlinear Abel fractional differential equation of the first kind
}

https://doi.org/10.1515/nleng-2018-0095

Received June 27, 2018; revised August 9, 2018; accepted September 30, 2018.

\begin{abstract}
Fractional calculus and fractional differential equations (FDE) have many applications in different branches of sciences. But, often a real nonlinear FDE has not the exact or analytical solution and must be solved numerically. Therefore, we aim to introduce a new numerical algorithm based on generalized Bessel function of the first kind (GBF), spectral methods and Newton-Krylov subspace method to solve nonlinear FDEs. In this paper, we use the GBFs as the basis functions. Then, we introduce explicit formulas to calculate Riemann-Liouville fractional integral and derivative of GBFs that are very helpful in computation and saving time. In the presented method, a nonlinear FDE will be converted to a nonlinear system of algebraic equations using collocation method based on GBF, then the solution of this nonlinear algebraic system will be achieved by using Newton-generalized minimum residual (Newton-Krylov) method. To illustrate the reliability and efficiency of the proposed method, we apply it to solve some examples of nonlinear Abel FDE.
\end{abstract}

Keywords: First kind of nonlinear Abel FDE; Generalized Bessel functions; Spectral collocation method; Nonlinear system of equations; Newton-Krylov methods

\section{Introduction}

Although fractional calculus is an ancient mathematical topic, however in the last few decades fractional calculus and fractional differential equations (FDEs) have found

\footnotetext{
*Corresponding Author: K. Parand, Department of Computer Sciences, Shahid Beheshti University, G.C., Tehran, Iran, Member of research group of Scientific Computing. E-mail: k_parand@sbu.ac.ir, Tel:+98 21 22431653; Fax:+98 2122431650

M. Nikarya, Department of Computer Sciences, Shahid Beheshti University, G.C., Tehran, Iran, E-mail: mehran.nikarya@gmail.com
}

many applications in physics, chemistry, engineering, finance, and other branches of sciences, therefore scientists are attracted to study FDEs more than ever; for instance, see [1-5]. Most results about solving nonlinear FDEs are obtained by using numerical methods, because only some particular FDEs can be solved analytically. Due to the growing applications, considerable attention has been given to the numerical solution of fractional differential equations (FDE) [6-10]. Previously, many of researchers study FDEs and attempt to solve them by utilizing several numerical techniques, for example, Alipour and Agahi by using new computational techniques based on $\mathrm{g}$ fractional differential operator [11], Wang and Fan by using the Chebyshev wavelet method[8], Rehman and Khan by using the Legendre wavelet method[9], Ordokhani and Rahimkhani by using Müntz-Legendre polynomials method[12], $\mathrm{Xu}$ et al. using an efficient quasi-Newton's method and simplified reproducing kernel method[13], Doha and Bhrawy by using the Tau method Chebyshev [14], Pezza and Pitolli by using a multi-scale collocation method [15], Esmaeili and Shamsi by using the pseudospectral method [16], Pedas and Tamme by using the spline collocation methods [17], as well as many other researchers by several methods have attemptet to solve linear or nonlinear FDEs [18-23, 23-26].

In this paper, we attempt to introduce a new method, based on a new class of Bessel functions namely GBFs and spectral collocation method for solving nonlinear Abel FDE of the first kind. Formerly, classical Bessel functions and Bessel polynomials have been used for solving several types of nonlinear problems [27-31]. Also previously, the classical Bessel function of the first kind has been used to solved nonlinear FDEs and fractional intrgro-differential equation, in their work the calculation of the fractional derivative of basis functions was not easy, for this reason they had to use few bases in spectral expansion [32].

Now, we aim to improve this problem, so we introduce the generalized Bessel function of the first kind (GBF), then by Riemann-Liouville fractional integral and derivative definition introduce explicit formulas to calculate fractional integro and derivative of GBF. Also, we introduce the 
derivative matrix of the Bessel function and GBF. Another novelty in this paper is using Newton-Krylov sub-space method to solve nonlinear system of algebraic equations that be obtained by spectral GBF method.

To show the reliability, efficiently and applicability of the proposed method we employ the presented method to solve nonlinear Abel FDE of the first kind [32, 33]:

$$
\begin{aligned}
& D^{\alpha} y(x)=a(x) y(x)^{3}+b(x) y(x)^{2}+c(x) y(x)+d(x), \\
& 0<\alpha<1, \quad y(0)=0,
\end{aligned}
$$

where $a(x), b(x), c(x)$ and $d(x)$ are functions of $x$, and $a(x) \neq 0 . D^{\alpha}$ is the fractional derivative operator. The Abel differential equation has a long history and therefore it can be easily found in many areas of pure mathematics and applied mathematics [33, 34]. Among the existing methods, the most efficient is an analytical method, thus, many scholars have applied various analytical methods to find a reliable solution for different mathematical problems [33, 35]. On the other hand, for many decades, nonlinear differential equations such as Able equation were and are in the center of attentions to find an applicable and reliable analytical solution for them. Abel equation appears to reduce the order of many higher orders nonlinear problems. Hence, every day, the exact solution is demanded for Abel differential equation in first and second kind[36, 37].

The remainder of this paper is organized as follows. In Section 1.1, some essential definitions and information about the fractional calculus theory and FDEs are described. The basic information of function approximation, Bessel function and generalized Bessel function, spectral collocation methods based on generalized Bessel functions and Newton-Krylov method are explained in Section 2. In section 3, some examples of fractional Abel equation are solved by the proposed method and their results are presented. Finally, in the last section, we have described several concluding remarks.

\subsection{Basic definitions of fractional calculus}

In this section, we present some notations, definitions and preliminary facts of the fractional calculus theory.

Definition 1. The Riemann-Liouville fractional integral operator $I^{\alpha}$ of order $\alpha$ on a usual Lebesgue space is given by

$$
I^{\alpha} f(t)=\frac{1}{\Gamma(\alpha)} \int_{0}^{t}(t-\tau)^{\alpha-1} f(\tau) d \tau .
$$

Some properties of this definition are:

$$
\begin{aligned}
& I^{0} f(t)=f(t) \\
& I^{\alpha} I^{\beta} f(t)=I^{\alpha+\beta} f(t) \\
& I^{\alpha} I^{\beta} f(t)=I^{\beta} I^{\alpha} f(t) \\
& I^{\alpha}(t)^{v}=\frac{\Gamma(v+1)}{\Gamma(\alpha+v+1)}(t)^{\alpha+v}
\end{aligned}
$$

where $f \in L_{1}[a, b], \beta, \alpha \geq 0$ and $v>-1$

Definition 2. The Riemann-Liouville fractional derivative of order $\alpha>0$ is defined as follows:

$D^{\alpha} f(t)=\frac{d^{m}}{d t^{m}}\left(I^{m-\alpha} f(t)\right)=\frac{1}{\Gamma(m-\alpha)} \frac{d^{m}}{d t^{m}} \int_{0}^{t}(t-\tau)^{m-\alpha-1} f(\tau) d \tau$

where $m$ is an integer, which is satisfied in $m-1<\alpha \leq$ $m$. Another fractional differential operator $D^{\alpha}$ proposed by Caputo.

\section{Spectral methods based on generalized Bessel functions}

Definition 3: Let $\Lambda=\{x \mid a<x<b\}$, then nonnegative function $\omega(x)$ is a weight function if:

- (a) $\omega(x) \geq 0$ is measurable on finite or infinite interval $\Lambda$.

- (b) $\mu_{k}=\int_{\Lambda} x^{k} \omega(x) d x<\infty$ for $k=0,1, \ldots$

- (c) $\int_{\Lambda} \omega(x) d x>0$.

Let $\omega$ is a certain weight function, therefore:

$$
L_{\omega}^{2}(\Lambda)=\left\{v \mid v \text { is measurable and }\|v\|_{\omega}<\infty\right\},
$$

where

$$
\|v\|_{\omega}=(v, v)_{\omega}^{1 / 2}=\left(\int_{\Lambda}|v(x)|^{2} \omega(x) d x\right)^{1 / 2} .
$$

In particular, in Hilbert space $L_{\omega}^{2}(\Lambda),(u, v)_{\omega}=$ $\int_{\Lambda} u(x) v(x) \omega(x) d x$ is inner product. If $\omega=1$ can be omitted, and let $\partial_{x}^{k} v=\frac{d^{k} v}{d x^{k}}$. Now for nonnegative integer $m$, we set

$$
H_{\omega}^{m}(\Lambda)=\left\{v \mid \partial_{x}^{k} v \in L_{\omega}^{2}(\Lambda), 0 \leq k \leq m\right\},
$$

with following norm, and semi-norm

$$
\begin{gathered}
(u, v)_{m, \omega}=\sum_{k=0}^{m}\left(\partial_{x}^{k} u, \partial_{x}^{k} v\right)_{\omega}, \quad|v|_{m, \omega}=\left\|\partial_{x}^{m} v\right\|_{\omega}, \\
\|v\|_{m, \omega}=(v, v)_{m, \omega}^{1 / 2} .
\end{gathered}
$$

For any real $r \geq 0$, we define the space $H_{\omega}^{r}(\Lambda)$ by the space interpolation as in Adams [38]. 


\subsection{Generalized Bessel function}

In this section, we explain Bessel function and generalized Bessel function of the first kind and some useful relations of them.The Bessel function of the first kind $J_{n}(x)$ is defined as follow:

$$
J_{n}(x)=\sum_{r=0}^{\infty} \frac{(-1)^{r}}{r ! \Gamma(n+r+1)}\left(\frac{x}{2}\right)^{2 r+n},
$$

where $\Gamma(\lambda)$ is the gamma function:

$$
\Gamma(\lambda)=\int_{0}^{\infty} e^{-t} t^{\lambda-1} d t .
$$

The series (4) is convergent for all $-\infty<x<\infty$. Actually, the Bessel function is a solution of the following SturmLiouville equation[39]:

$$
\begin{aligned}
& x^{2} y^{\prime \prime}(x)+x y^{\prime}(x)+\left(x^{2}-n^{2}\right) y(x)=0, \\
& \text { for } x \in(-\infty, \infty), \quad(n \in \mathbb{R}) .
\end{aligned}
$$

It is clear that, for integer $n, J_{n}(x)$ are linear independent. Some recursive relations of Bessel functions are as follows [39]:

$$
\begin{aligned}
& \frac{d}{d x}\left(x^{n} J_{n}(x)\right)=x^{n} J_{n-1}(x), \\
& J_{n}^{\prime}(x)=J_{n-1}(x)-\frac{n}{x} J_{n}(x), \\
& J_{n}^{\prime}(x)=\frac{n}{x} J_{n}(x)-J_{n+1}(x),
\end{aligned}
$$

Lemma: One of the useful recursion relations of Bessel function of the first kind is:

$$
J_{n}^{\prime}(x)=\frac{1}{2} J_{n-1}(x)-\frac{1}{2} J_{n+1}(x) .
$$

Proof. By deriving of (4) and using expansions of $J_{n-1}(x)$ and $J_{n+1}(x)$, the result is desirable.

Remark: The derivative operational matrix of the first kind Bessel functions can be obtained as follows:

Let $J_{n}=\left[J_{0}(x), J_{1}(x), J_{2}(x), \ldots, J_{n}(x)\right]^{T}$ therefor $J^{\prime}=D J_{n}$, where $D$ is derivative operational matrix and is obtained by (6):

$$
D=\left[\begin{array}{ccccccc}
0 & -1 & 0 & 0 & 0 & \cdots & 0 \\
\frac{1}{2} & 0 & -\frac{1}{2} & 0 & 0 & \cdots & 0 \\
0 & \frac{1}{2} & 0 & -\frac{1}{2} & 0 & \cdots & 0 \\
0 & 0 & \frac{1}{2} & 0 & -\frac{1}{2} & \cdots & 0 \\
\vdots & \ddots & & \ddots & & \ddots & \\
a_{0} & a_{2} & a_{3} & \cdots & \cdots & 0 & a_{n}
\end{array}\right]
$$

where the $a_{0}, a_{1}, a_{2}, \ldots, a_{n}$ will be obtained by an interpolation technique.
Now we define generalized Bessel function (GBF) of the first kind as follows:

Definition 4: Let $n>-1$ then generalized Bessel function (GBF) of the first kind is defined as:

$$
\widehat{J}_{n}(x)=x^{\frac{n}{2}} J_{n}(\sqrt{x}) .
$$

Theorem 1: A recursive relation of derivative of GBF of the first kind is as follows,

$$
\frac{d}{d x} \widehat{J}_{n}(x)=\frac{1}{2} \widehat{J}_{n-1}(x)
$$

Proof. By using GBF of the first kind definition and expansion of $J_{n}(x)$ the result will be achieved.

Remark: the derivative operational matrix of the first kind GBF can be obtained as follow:

Let $\widehat{J}_{n}=\left[\widehat{J}_{0}(x), \widehat{J}_{1}(x), \widehat{J}_{2}(x), \ldots, \widehat{J}_{n}(x)\right]^{T}$ therefor $\widehat{J}_{n}^{\prime}=\widehat{D} \widehat{J}_{n}$, where $\widehat{D}$ is derivative operational matrix and is obtained using (9):

$$
D=\left[\begin{array}{ccccccc}
b_{0} & b_{1} & b_{2} & \cdots & \cdots & b_{n-1} & b_{n} \\
\frac{1}{2} & 0 & 0 & 0 & 0 & \cdots & 0 \\
0 & \frac{1}{2} & 0 & 0 & 0 & \cdots & 0 \\
0 & 0 & \frac{1}{2} & 0 & 0 & \cdots & 0 \\
\vdots & \ddots & & \ddots & & \ddots & \\
0 & 0 & 0 & \cdots & \cdots & \frac{1}{2} & 0
\end{array}\right]
$$

where the $b_{0}, b_{1}, b_{2}, \ldots, b_{n}$ will be obtained by an interpolation technique. Notice that:

$$
\widehat{J}_{-1}(x)=\frac{-\widehat{J}_{1}(x)}{x}
$$

Theorem 2: If $\alpha>0$ and $n>-1$ then the RiemannLiouville fractional integral of generalized Bessel function of the first kind is:

$$
I^{\alpha} \widehat{J}_{n}(x)=2^{\alpha} \widehat{J}_{n+\alpha}(x),
$$

and the Riemann-Liouville fractional derivative of GBF is:

$$
D^{\alpha} \widehat{J}_{n}(x)=2^{-\alpha} \widehat{J}_{n-\alpha}(x) .
$$

Proof. By using definition of Bessel function of the first kind (4) and Riemann-Liouville fractional integral of $\widehat{J}_{n}(x)$, we can write:

$$
\begin{aligned}
I^{\alpha} \widehat{J}_{n}(x) & =I^{\alpha}\left[x^{\frac{n}{2}} J_{n}(\sqrt{x})\right]=I^{\alpha}\left[x^{\frac{n}{2}} \sum_{r=0}^{\infty} \frac{(-1)^{r}}{r ! \Gamma(n+r+1)}\left(\frac{\sqrt{x}}{2}\right)^{2 r+n}\right] \\
& =I^{\alpha}\left[\sum_{r=0}^{\infty} \frac{(-1)^{r}}{r ! \Gamma(n+r+1)} \frac{x^{r+n}}{2^{2 r+n}}\right] \\
& =\frac{1}{\Gamma(\alpha)} \int_{0}^{x}(x-t)^{\alpha-1} \sum_{r=0}^{\infty} \frac{(-1)^{r}}{r ! \Gamma(n+r+1)} \frac{t^{r+n}}{2^{2 r+n}} d t
\end{aligned}
$$


by calculating and relations of Gamma function for real values, we have:

$$
I^{\alpha} \widehat{J}_{n}(x)=\sum_{r=0}^{\infty} \frac{(-1)^{r}}{r ! \Gamma(n+r+\alpha+1)} \frac{x^{r+n+\alpha}}{2^{2 r+n}} .
$$

Which ultimately results

$$
I^{\alpha} \widehat{J}_{n}(x)=2^{\alpha} \sum_{r=0}^{\infty} \frac{(-1)^{r}}{r ! \Gamma(n+r+\alpha+1)} \frac{x^{r+n+\alpha}}{2^{2 r+n+\alpha}}=2^{\alpha} \widehat{J}_{n+\alpha}(x) .
$$

Also, the Equation (12) can be concluded immediately in the same way.

To comfort we denominate:

$$
\widehat{J}_{n}^{\alpha}(x)=2^{-\alpha} \widehat{J}_{n-\alpha}(x)=D^{\alpha} \widehat{J}_{n}(x),
$$

and $\left.\widehat{J}_{n}^{\alpha}=\widehat{J}_{0}^{\alpha}(x), \widehat{J}_{1}^{\alpha}(x), \ldots, \widehat{J}_{n}^{\alpha}(x)\right]^{T}$.

In this paper, we use the GBFs as the basis functions of $L^{2}(\Lambda)$. Let $N$ be a positive integer, we define the following space:

$$
J_{N}=\operatorname{span}\left\{\widehat{J}_{0}, \widehat{J}_{1}, \ldots, \widehat{J}_{N}\right\},
$$

this is clear that $\mathcal{J}_{N}(\Lambda) \subseteq H^{r}(\Lambda) \subseteq L^{2}(\Lambda)$.

Now consider $L^{2}(\Lambda)$-orthogonal projection $P_{N}: L^{2}(\Lambda) \rightarrow$ $J_{N}$ that, for any $v \in L^{2}(\Lambda)$ :

$$
\left(P_{N} v-v, \phi\right)=0, \quad \forall \phi \in \mathcal{J}_{N} .
$$

or equivalently

$$
P_{N} v(x)=\sum_{i=0}^{N} \hat{v}_{i} J_{i}(x),
$$

In other words, let $y$ be an arbitrary element in $L^{2}(\Lambda)$, since $J_{N}$ is finite dimensional subspace of $L^{2}, y$ has a unique best approximation $y_{0} \in \mathcal{J}_{N}$ such that

$$
\forall v \in \mathcal{J}_{N}, \quad\left\|y-y_{0}\right\| \leq\|y-v\|,
$$

so, for any $v \in H^{r}(\Lambda)$ and $r \geq 0$ we have[40]:

$$
\left\|P_{N} v-v\right\| \leq c N^{-r}\|v\|_{r}
$$

Hence, the error of this approximation by increasing $N$ will be decreased, in numerical examples, this principle will be shown.

\subsection{Collocation method}

Spectral methods, in the context of numerical schemes for solving differential equations, generically belong to the family of weighted residual methods (WRMs) [41-43].
WRMs represent a particular group of approximation techniques, in which the residuals (or errors) are minimized in a certain way and thereby leading to specific methods including Galerkin, Petrov-Galerkin, collocation and tau formulations. consider the approximation of the following problem via spectral method:

$$
\mathcal{L} u(x)+\mathcal{P}^{\alpha} u(x)+\mathcal{N} u(x)=f(x) \quad, \quad(x) \in \Omega,
$$

where $\mathcal{L}$ is the differential or integral operator, $\mathcal{P}^{\alpha}$ is fractional operator (differential or integral fractional operator), $\mathcal{N}$ is a lower-order linear and/or nonlinear operator involving only derivatives (if exist) and $f(x)$ is a function of variables $x$, with enough initial and boundary conditions. The starting point of the spectral methods is to approximate the solution $u(x) \in H^{r}(\Omega)$ by a finite summation:

$$
u(x) \approx u_{N}(x)=\sum_{i=0}^{N} a_{i} \phi_{i}(x), \quad x \in \Omega,
$$

where $\phi_{n}$ 's are the basis functions that we have chosen $\widehat{J}_{n}(x)$ as the basis function and the expansion's coefficients must be determined. Substituting $u$ with $u_{N}$ in (17) leads to the residual function:

$$
\mathcal{R}_{N}(x)=\mathcal{L} u_{N}(x)+\mathcal{P}^{\alpha} u_{N}(x)+\mathcal{N} u_{N}(x)-f(x) .
$$

Notice that, $\mathcal{P}^{\alpha} u_{N}(x)$ is calculated by (12) and (15):

$$
\mathcal{P}^{\alpha} u_{N}(x)=\sum_{i=0}^{N} a_{i} \widehat{J}_{n}^{\alpha}(x) .
$$

The notion of the WRM is to force the residual function to zero in adequate norm by requiring:

$$
\begin{aligned}
& <\mathcal{R}_{N}, \psi_{i}>_{\omega}=0, \quad 0 \leq i \leq N \\
& \equiv \int_{\Omega} \mathcal{R}_{N}(x) \psi_{i}(x) \omega(x) d x=0, \quad 0 \leq i \leq N
\end{aligned}
$$

where $\left\{\psi_{k}\right\}$ are test functions, and $\omega$ is a positive weight function. The choice of test functions results to a kind of the spectral methods $[41,44]$. A method for forcing the residual function (19) to zero, is the collocation algorithm $[27,44,45]$. In this method, by choosing Lagrange basis polynomials as test function, such that $\psi_{j}(x)=L_{j}(x)$ and using Gauss quadrature rule in (20) we can write[41]:

$$
\begin{gathered}
<\mathcal{R}_{N}, \psi_{i}>\omega=\sum_{j=0}^{N} \mathcal{R}_{N}\left(x_{j}\right) \psi_{i}\left(x_{j}\right) \omega\left(x_{j}\right) W_{q}=0 . \\
0 \leq i, j \leq N .
\end{gathered}
$$

Where $x_{j}$ is Gauss points and $W_{j}$ is Gauss weights. If we use $x_{j}$ as collocation points and construct Lagrangian polynomial based on these points, according to Lagrange polynomials definition $\psi_{i}\left(x_{j}\right)=\delta_{i j}$ and by choosing $\omega=1$ :

$$
\mathcal{R}_{N}\left(x_{j}\right)=0, \quad j=0,1, \ldots, N .
$$


In this paper, since the FDEs (17) are nonlinear, the obtained system of equations (22) is nonlinear, too. To solve this nonlinear algebraic system, we use NewtonKrylov sub-space method [46, 47]. In this method for solving a nonlinear system of algebraic equations $F(x)=$ 0 , where $F: \mathbb{R}^{n} \rightarrow \mathbb{R}^{n}$ is a function $F(x)=$ $\left(f_{1}(x), f_{2}(x), f_{3}(x), \ldots, f_{n}(x)\right)^{T}$ and $x \in \mathbb{R}^{n}$ is a vector. Speed and the accuracy of solving this nonlinear system are very important. Many works have been done to improve. One of the best methods to solve a nonlinear system is classical Newton's iterative method:

$$
F\left(x_{n+1}\right)=F\left(x_{n}\right)+\left(x_{n}-x_{n+1}\right) F^{\prime}\left(x_{n}\right),
$$

where $F^{\prime}(x)=J(x)$ is the $n \times n$ Jacobian matrix. Therefore:

$$
x_{n+1}=x_{n}-J\left(x_{n}\right)^{-1} F\left(x_{n}\right) .
$$

In fact, in each iteration, a linear system must be solved:

$$
\left\{\begin{array}{l}
x_{n+1}=x_{n}+\delta x_{n} \\
J\left(x_{n}\right) \delta x_{n}=F\left(x_{n}\right) .
\end{array}\right.
$$

Now, we use the generalized minimum residual method to solve obtained linear system in each Newton iteration. We have explained this method in previous work $[46,47]$.

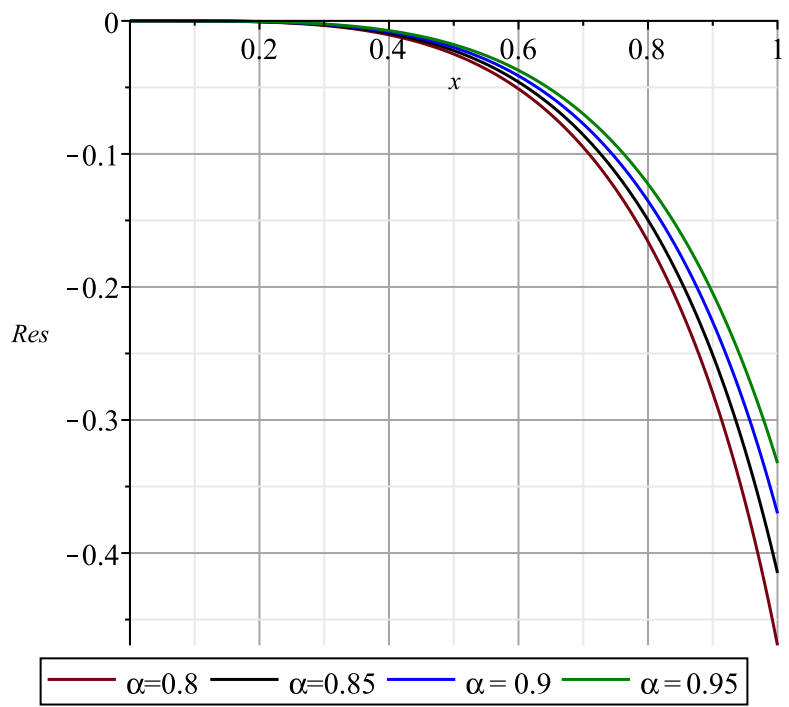

Fig. 1: The graphs of the approximation solutions of Example 1 for $N=30$ and $\alpha=0.8,0.85,0.9,0.95$.

\section{Solving Some example of nonlinear Abel fractional differential equation of the first kind}

In this section, we apply the proposed method which is described in section 2.2, to solve some examples of nonlinear Abel fractional differential equation of the first kind (1). All examples that are chosen to solve in this section have not analytical and semi-analytical solution.

In the all of the following examples, we use the roots of shifted Chebyshev polynomials as the collocation points, the initial guess of Newton-Krylov method is vector $[1,1, \ldots, 1]^{T}$ and numbers of Newton-Krylov iterations maximum is 10 .

Now, we apply the proposed method to solve equation (1) with initial condition $y(0)=0$ in general form. To do this, we approximate the $y(x)$ as follow:

$$
y(x) \approx y_{N}(x)=\sum_{i=1}^{N} a_{i} \widehat{J}_{i}(x) \widehat{J}_{N},
$$

then we construct the residual function as follows:

$$
\begin{aligned}
\operatorname{Res}_{N}(x)= & D^{\alpha} y_{N}(x)-y_{N}(x)^{3} \sin (x) \\
& +x y_{N}(x)^{2}-x^{2} y_{N}(x)+x^{3}
\end{aligned}
$$

According to collocation technique, $N$ nonlinear equation will be obtained via (20)-(22), then by solving this nonlinear system of equations by using Newton-Krylov the approximation solution $y_{N}(x)$ be achieved.

Example 1. Consider the fist example of nonlinear Abel fractional differential equation of the first kind [32, 33]:

$$
\begin{aligned}
& D^{\alpha} y(x)=y(x)^{3} \sin (x)-x y(x)^{2}+x^{2} y(x)-x^{3}, \\
& \quad x \in(0,1], \quad \alpha \in(0,1)
\end{aligned}
$$

with the initial condition:

$$
y(0)=0 .
$$

This example has been solved by $\mathrm{Xu}$ and He by using the short memory principle (SMP) [33]. Also, Parand and Nikarya have solved this example by using a collocation method based on classical Bessel function [32].

Now, we have solved this example by employing the proposed method for $\alpha=0.8,0.85,0.9$ and 0.95. The obtained graphs of approximation solution $y_{N}(x)$ of Eq. (27) are shown in Fig. 1, also in Table 1 the values of $y_{N}(x)$ and $\operatorname{Res}(x)$ is compared with results of BFC method [32] and orthogonal fractional polynomials (OFP) method[48] 


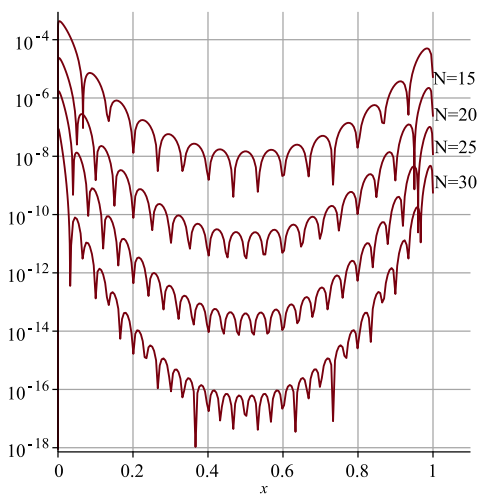

(a) $\alpha=0.8$

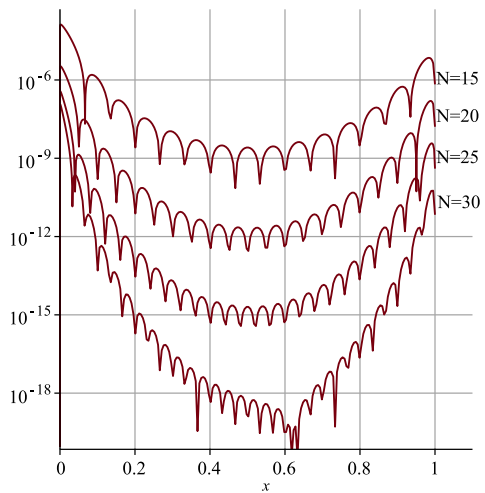

(c) $\alpha=0.90$

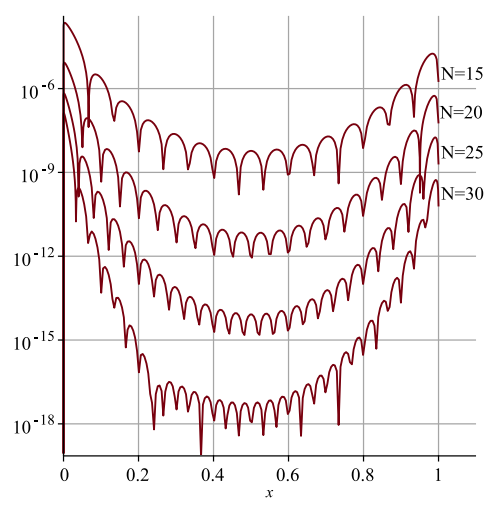

(b) $\alpha=0.85$

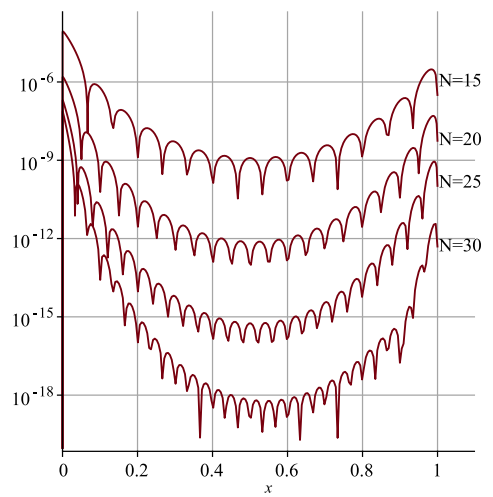

(d) $\alpha=0.95$

Fig. 2: The graphs of residual function to show convergence rate of the proposed method for solving Example 1 for $\alpha=0.8,0.85,0.9,0.95$ and several $N$.

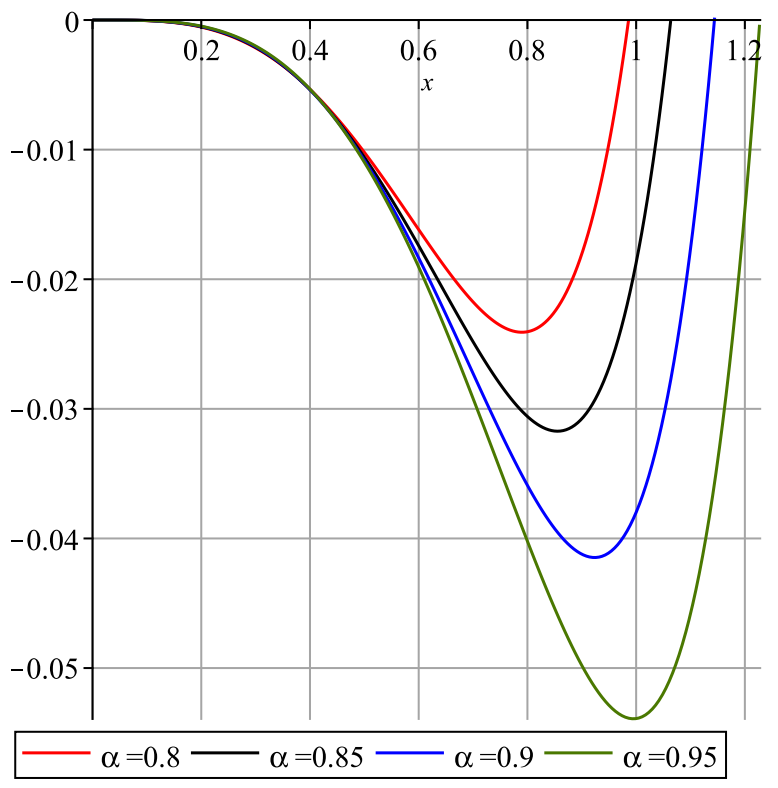

Fig. 3: The graphs of the approximation solutions of Example 2 for $\alpha=0.8,0.85,0.9,0.95$ and $N=30$. for several $\alpha$. To show the accuracy and convergency of the present method to solve this example for $\alpha=$ $0.8,0.85,0.9$ and 0.95 , the graphs of residual function $\operatorname{Res}(x)$ is presented for several $N$ in Fig. 2, in this these graphs, we showed that, by increasing the $N$ the residual function decreases, to show the convergence of the presented method.

Example 2. Consider the first kind Abel fractional differential equation:

$$
D^{\alpha} y(x)=e^{-x} y(x)^{3}-\frac{y(x)^{2}}{\sin (x)}+x^{2} y(x)-x^{3} \tan (\alpha-x)
$$

with initial conditions:

$$
y(0)=0 .
$$

We have solved Eq. (29) by the proposed method for $\alpha=$ $0.8,0.85,0.9$ and 0.95 . The graphs of approximation solution $y_{N}(x)$ are shown in Fig. 3, in Table 2 the values of $y_{N}(x)$ is presented for $\alpha=0.8,0.85,0.9$ and 0.95 . Also to show the accuracy and convergency of the present method to solve this example for $\alpha=0.8,0.85,0.9$ and 0.95 , the graphs of residual function $\operatorname{Res}(x)$ is presented for several 
Table 1: Comparison of obtained values of the proposed method and methods of $[32,48]$ for Example 1 for $\alpha=0.8,0.85,0.9$

\begin{tabular}{|c|c|c|c|c|c|c|}
\hline \multirow[b]{2}{*}{$x$} & \multicolumn{2}{|c|}{ Proposed method } & \multicolumn{2}{|c|}{ OFP method [48] } & \multicolumn{2}{|c|}{ BFC method[32] } \\
\hline & $y_{N}(x)$ & $\operatorname{Res}(x)$ & $y_{N}(x)$ & $\operatorname{Res}(x)$ & $y_{N}(x)$ & $\operatorname{Res}(x)$ \\
\hline & $\alpha=0.8$ & & & & & \\
\hline 0.2 & -0.000744342596 & $5.21 e-16$ & $-7.443 e-4$ & $4.39 e-8$ & -0.000744347 & $2.34 e-7$ \\
\hline 0.4 & -0.010522091334 & $1.41 e-18$ & $-1.052 e-2$ & $3.00 e-8$ & -0.010522097 & $8.25 e-8$ \\
\hline 0.6 & -0.051085883734 & $2.12 e-18$ & $-5.108 e-2$ & $2.06 e-8$ & -0.051085888 & $4.23 e-7$ \\
\hline 0.8 & -0.165925971799 & $4.84 e-16$ & $-1.659 e-1$ & $4.53 e-8$ & -0.165925912 & $1.00 e-6$ \\
\hline \multirow[t]{2}{*}{1.0} & -0.469342130398 & $5.29 e-11$ & $-4.693 e-1$ & $7.88 e-8$ & -0.469341539 & $8.71 e-5$ \\
\hline & $\alpha=0.85$ & & & & & \\
\hline 0.2 & -0.000637969398 & $5.26 e-18$ & & & -0.000637325 & $5.21 e-6$ \\
\hline 0.4 & -0.009317385395 & $1.02 e-19$ & & & -0.009316125 & $7.16 e-6$ \\
\hline 0.6 & -0.045936456944 & $2.19 e-19$ & & & -0.045937147 & $9.43 e-6$ \\
\hline 0.8 & -0.149705797239 & $5.53 e-17$ & & & -0.149708698 & $5.18 e-5$ \\
\hline \multirow[t]{2}{*}{1.0} & -0.414921134187 & $6.04 e-11$ & & & -0.414919988 & $4.36 e-4$ \\
\hline & $\alpha=0.9$ & & & & & \\
\hline 0.2 & -0.000546521537 & $1.41 e-17$ & $-5.465 e-4$ & $1.66 e-09$ & -0.0005465031 & $3.26 e-6$ \\
\hline 0.4 & -0.008248714922 & $3.63 e-20$ & $-8.248 e-3$ & $1.06 e-09$ & -0.0082482782 & $4.28 e-6$ \\
\hline 0.6 & -0.041322824126 & $2.82 e-21$ & $-4.132 e-2$ & $9.61 e-10$ & -0.0413235146 & $8.33 e-6$ \\
\hline 0.8 & -0.135349790084 & $4.30 e-18$ & $-1.353 e-1$ & $9.78 e-10$ & -0.1353508143 & $2.90 e-5$ \\
\hline 1.0 & -0.370165019409 & $6.84 e-12$ & $-3.701 e-1$ & $1.49 e-09$ & -0.3701646146 & $2.31 e-4$ \\
\hline
\end{tabular}
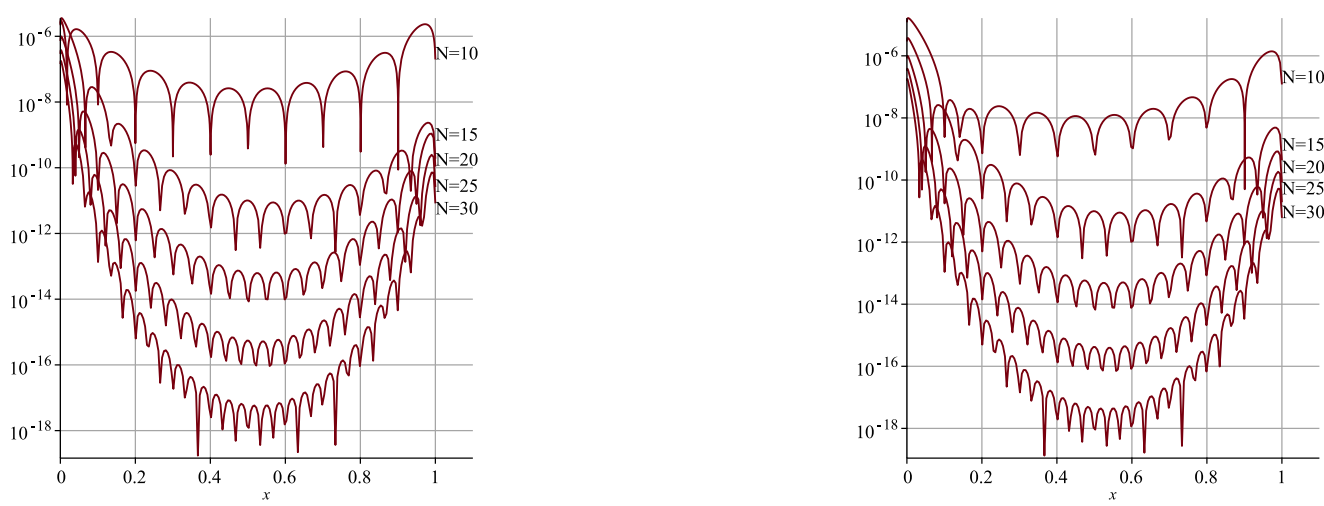

(a) $\alpha=0.8$

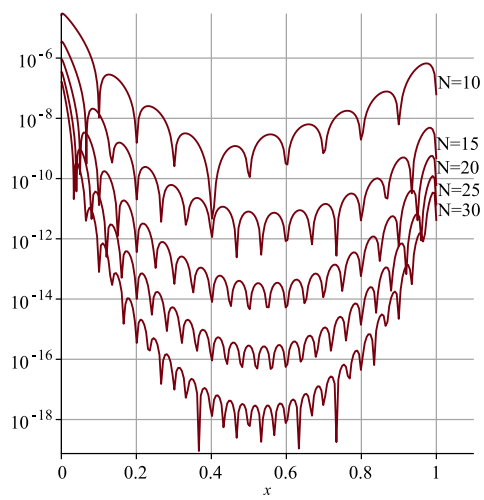

(b) $\alpha=0.85$

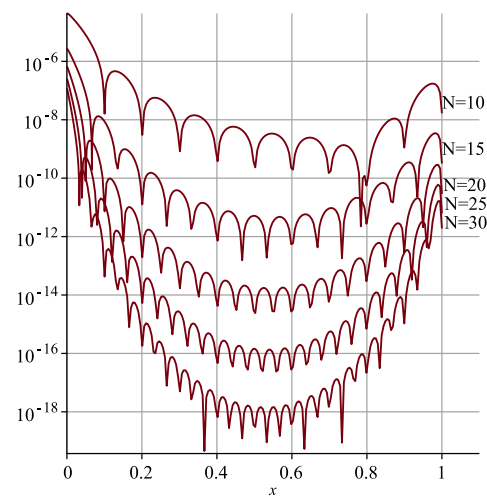

(c) $\alpha=0.90$

(d) $\alpha=0.95$

Fig. 4: The graphs of residual function to show convergence rate of the proposed method for solving Example. 2 for $\alpha=0.8,0.85,0.9,0.95$ and several $N$. 
Table 2: The values of approximation solution $y_{N}(x)$ of Example 2 for $\alpha=0.8,0.85,0.9,0.95$ and $N=30$

\begin{tabular}{lllll} 
& $\alpha=0.8$ & $\alpha=0.85$ & $\alpha=0.9$ & $\alpha=0.95$ \\
\hline 0.2 & -0.0005475041 & -0.0005222619 & -0.0004968084 & -0.0004718497 \\
0.4 & -0.0053435740 & -0.0053795562 & -0.0053722684 & -0.0053332339 \\
0.6 & -0.0161724534 & -0.0174150949 & -0.0183578125 & -0.0190602822 \\
0.8 & -0.0240508103 & -0.0305890412 & -0.0359020939 & -0.0402140291 \\
1.0 & 0.00457208778 & -0.0187336581 & -0.0379851769 & -0.0538981175 \\
\hline
\end{tabular}

Table 3: The values of approximate solution $y_{N}(x)$ of Example 3 for $\alpha=0.8,0.85,0.9,0.95$ and $N=30$

\begin{tabular}{l|cccc}
\hline$x$ & $\alpha=0.8$ & $\alpha=0.85$ & $\alpha=0.9$ & $\alpha=0.95$ \\
\hline 0.2 & 0.03363616357 & 0.02961943104 & 0.02608193866 & 0.022960828432 \\
0.4 & 0.12181916687 & 0.11053132512 & 0.10037152807 & 0.09118563054 \\
0.6 & 0.26234938310 & 0.24253149973 & 0.22437891679 & 0.20768345736 \\
0.8 & 0.44042831745 & 0.41646752645 & 0.39326700741 & 0.37093250960 \\
1 & 0.59212471706 & 0.57961602578 & 0.56516743215 & 0.54897123418 \\
\hline
\end{tabular}

Table 4: The values of approximate solution $y_{N}(x)$ of Example 4 for $\alpha=0.85,0.9,0.95, N=30$ and $\alpha=0.8, N=120$

\begin{tabular}{l|cccc}
\hline$x$ & $\alpha=0.8$ & $\alpha=0.85$ & $\alpha=0.9$ & $\alpha=0.95$ \\
\hline 0.1 & 0.0096441004 & 0.0082085561 & 0.0069957467 & 0.0059478742 \\
0.3 & 0.0757064787 & 0.0674889644 & 0.0602060179 & 0.0537146181 \\
0.5 & 0.2157562378 & 0.1950112085 & 0.1766105697 & 0.1601707949 \\
0.7 & 0.4861687659 & 0.4365854896 & 0.3945279816 & 0.3581641713 \\
0.9 & 1.2377342889 & 1.0106236135 & 0.8650944099 & 0.7594334013 \\
\hline
\end{tabular}

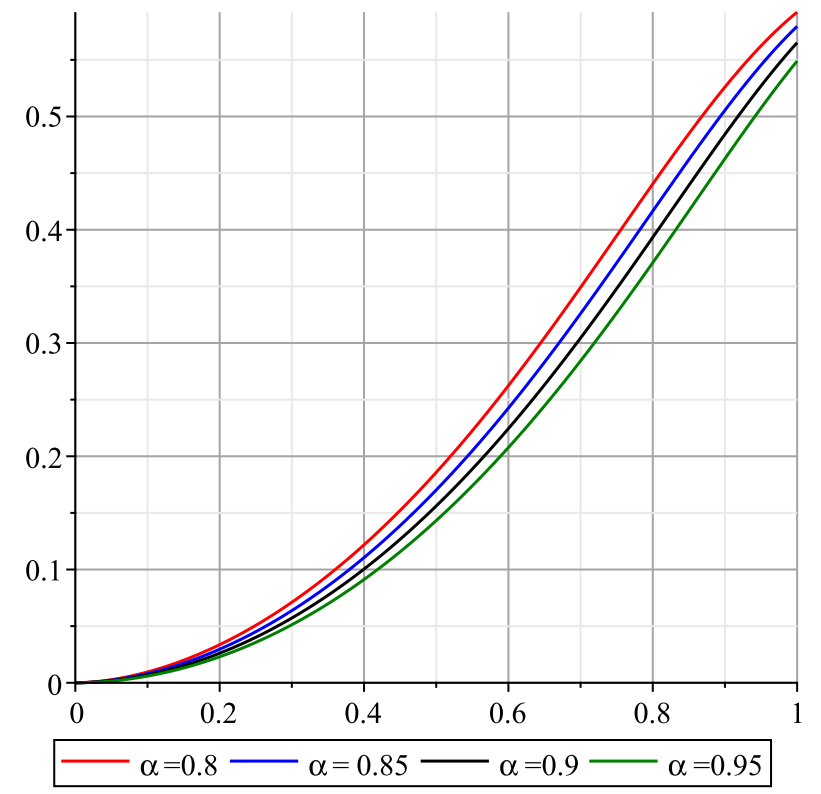

Fig. 5: The graphs of the approximation solutions of Example 3 for $N=30$ and $\alpha=0.8,0.85,0.9,0.95$ and $N=30$.

$N$ in Fig. 4, in this these graphs, we showed that, by increasing the $N$ the residual function decreases.
Example 3. Consider the fractional differential equation:

$$
D^{\alpha} y(x)=-\frac{y(x)^{3}}{\cos ^{2}(x)}+\frac{y(x)^{2}}{\sin (x)}-x^{2} y(x)+\tan (x)
$$

with conditions:

$$
y(0)=0 .
$$

We have solved Eq. (31) by the proposed method for $\alpha=$ $0.8,0.85,0.9$ and 0.95 . The graphs of obtained approximation solution $y_{N}(x)$ are shown in Fig. 5. Table 3 shows the values of $y_{N}(x)$ for $\alpha=0.8,0.85,0.9$ and 0.95 . Also to show the accuracy and convergency of the present method to solve this example for $\alpha=0.8,0.85,0.9$ and 0.95 , the graphs of residual function $\operatorname{Res}(x)$ is presented for several $N$ in Fig. 6, in this these graphs, we showed that, by increasing the $N$ the residual function decreases.

Example 4. Consider the fractional differential equation:

$$
D^{\alpha} y(x)=x^{2} y(x)^{3}+x y(x)^{2}+y(x) \sqrt{x}+\tan (x)
$$

with the initial condition:

$$
y(0)=0 .
$$

This example has not the exact or analytical solution, too. This example has been solved by $\mathrm{Xu}$ and He by using the short memory principle (SMP) [33].

We have solved Eq. (33) by the proposed method for $\alpha=$ 


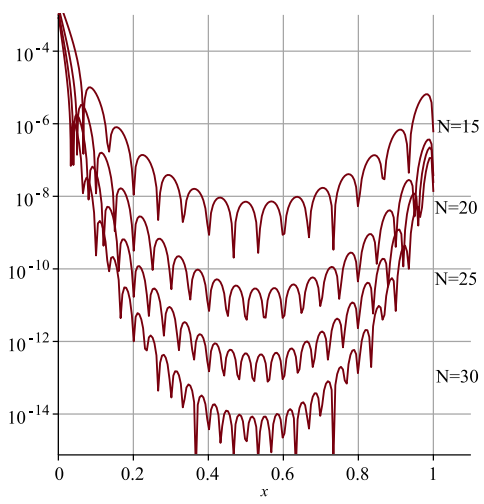

(a) $\alpha=0.8$

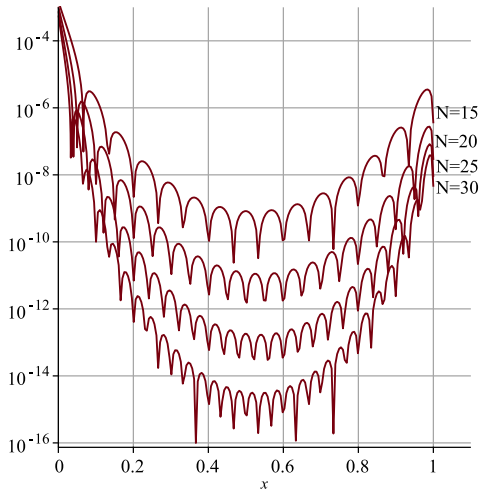

(c) $\alpha=0.90$

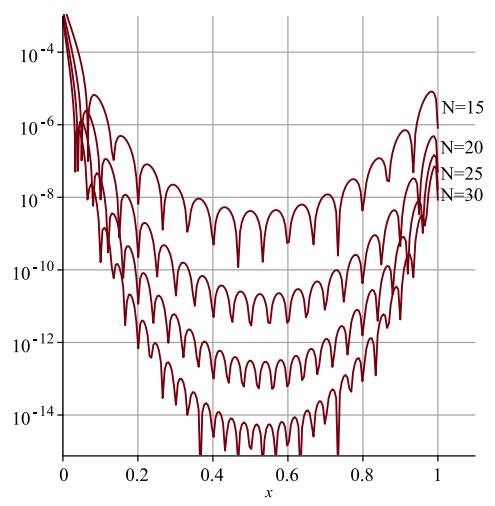

(b) $\alpha=0.85$

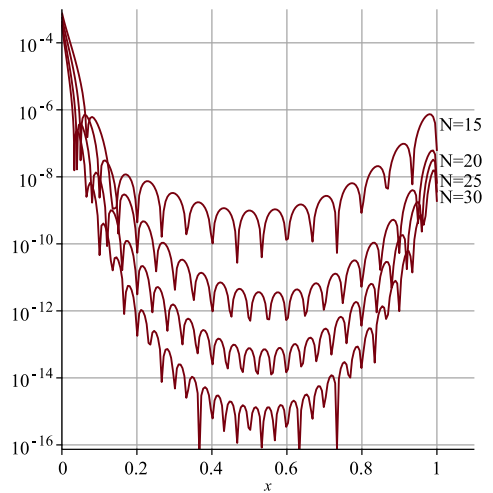

(d) $\alpha=0.95$

Fig. 6: The graphs of residual function to show convergence rate of the proposed method for solving Example 3 for $\alpha=0.8,0.85,0.9,0.95$ and several $N$

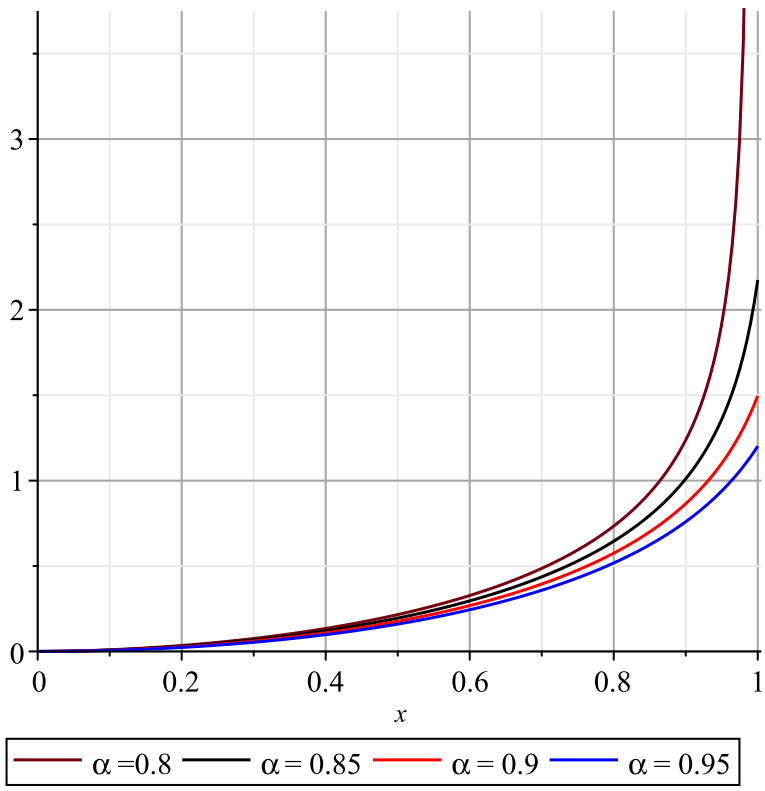

Fig. 7: The graphs of the approximation solutions of Example 4 for $\alpha=0.8,0.85,0.9,95$ and $N=30$.
$0.8,0.85,0.9$ and 0.95 . The graphs of the obtained approximation solution $y_{N}(x)$ are shown in Fig. 7. Table 4 shows the values of $y_{N}(x)$ for $\alpha=0.8,0.85,0.9$ and 0.95 . Also to show the accuracy and convergency of the present method to solve this example for $\alpha=0.8,0.85,0.9$ and 0.95 , the graphs of residual function $\operatorname{Res}(x)$ is presented for several $N$ in Fig. 8, in this these graphs, we showed that, by increasing the $N$ the residual function decreases.

\section{Conclusion}

The fractional calculus and fractional differential equations have found application in different sciences. But real and practical FDEs often have not exact or analytical solution, therefore, numerical solving of the fractional differential equations have become an attractive field of applied mathematics and computer science. In this article, we have introduced a new and high accurate numerical method based on generalized Bessel function (GBF) and collocation method to solve Abel fractional differential 


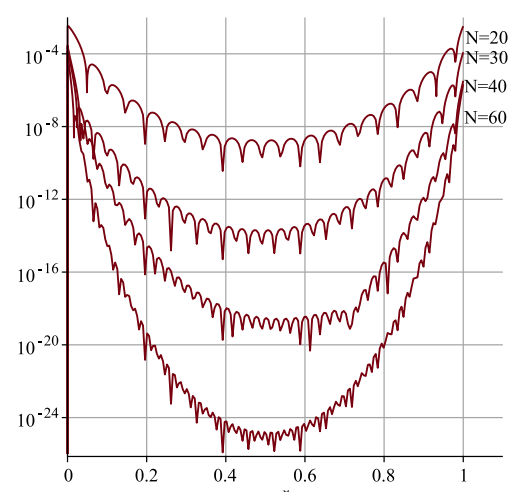

(a) $\alpha=0.8$

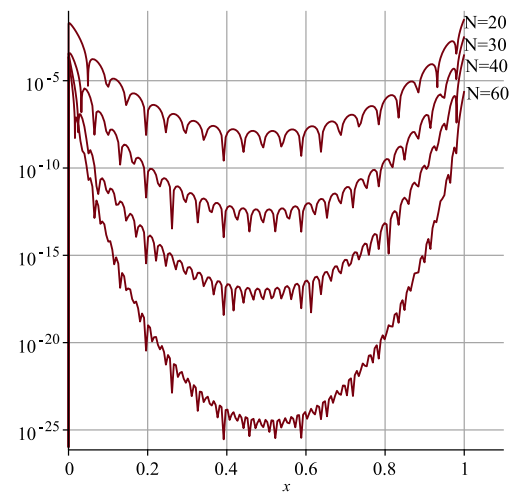

(c) $\alpha=0.90$

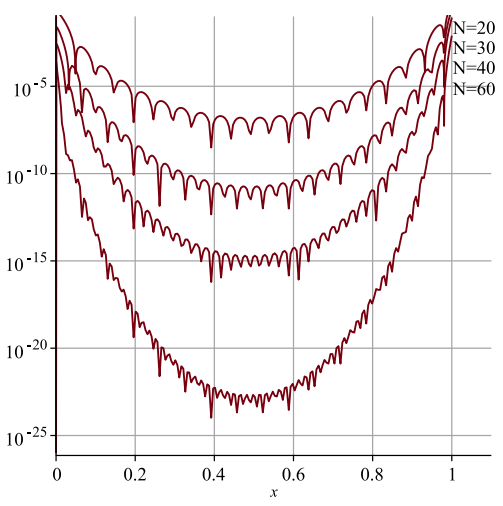

(b) $\alpha=0.85$

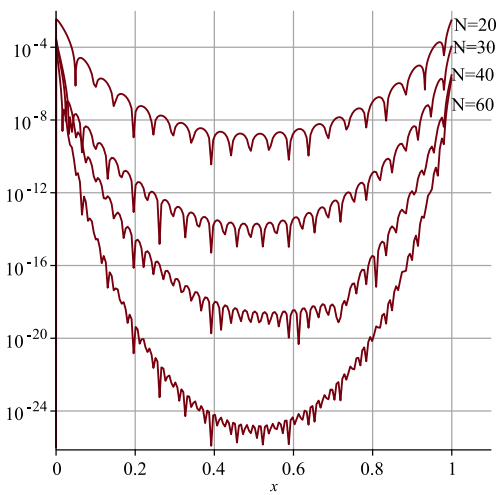

Fig. 8: The graphs of residual function to show convergence rate of the proposed method for solving Example 4 for $\alpha=0.8,0.85,0.9,0.95$ and several $N$

equation of the first kind. Also, in this paper, we have introduced an explicit formula to calculate Riemann-Liouville fractional derivative and integral of GBF, this formula causes the calculations be easier and faster. In the proposed method to solve obtained nonlinear algebraic systems, we use Newton-Krylov sub-space method. In this paper, we have solved the nonlinear Abel FDE of the first kind, that has not the exact or analytical solution. In this paper, we show the applicability and reliability of the proposed method to solve nonlinear FDEs.

Acknowledgement: The corresponding author would like to thank Shahid Beheshti University for the awarded grant.

\section{References}

[1] J. T. Machado, V. Kiryakova, F. Mainardi, Recent history of fractional calculus, Commun. Nonlinear Sci. Numer. Simul. 3 (2011) 1140-1753.

[2] K. Oldham, Fractional differential equations in electrochemistry, Adv. Eng. Soft. 41 (2010) 9-17.
[3] R. Hilfer, Applications of fractional calculus in physics, World Scientific Publishing Company, Singapore, 2000.

[4] K. Diethelm, The Analysis of Fractional Differential Equations, Springer-Verlag, Berlin Heidelberg, 2010.

[5] L. Suarez, A. Shokooh, An eigenvector expansion method for the solution of motion containing fractional derivatives, J. Appl. Mech. 64 (1997) 629-675.

[6] Z. Odibat, S. Momani, Numerical methods for nonlinear partial differential equations of fractional order, Appl. Math. Model. 32 (2008) 28-39.

[7] S. Momani, R. Qaralleh, Numerical approximations and Padé approximats for a fractional population growth model, Appl. Math. Model. 31 (2007) 1907-1914.

[8] Y. Wang, Q. Fan, The second kind Chebyshev wavelet method for solving fractional differential equations, Appl. Math. Comput. 218 (2011) 8592-8601.

[9] M. Rehman, R. A.Khan, The Legendre wavelet method for solving fractional differential equations, Commun. Nonlinear Sci. Numer. Simul. 16 (2011) 4163-4173.

[10] A. Saadatmandi, M. Dehghan, A new operational matrix for solving fractional-order differential equations, Comp. Math. Appl. 59 (2010) 1326-1336.

[11] M. Alipour, H. Agahi, New computational techniques for solving nonlinear problems using g-fractional differential operator, J. Comput. Appl. Math. 330 (2018) 70 - 74. 
[12] Y. Ordokhani, P. Rahimkhani, A numerical technique for solving fractional variational problems by müntz-legendre polynomials, J. Appl. Math. Comput. 58 (2018) 75-94.

[13] M. Xu, J. Niu, Y. Lin, An efficient method for fractional nonlinear differential equations by quasi-Newton's method and simplified reproducing kernel method, Math. Methods Appl. Sci. 41 (2017) 5-14.

[14] E. Doha, A. Bhrawy, S. Ezz-Eldien, Efficient Chebyshev spectral methods for solving multi-term fractional orders differential equations, Appl. Math. Model. 36 (2011) 5662-5672.

[15] L. Pezza, F. Pitolli, A multiscale collocation method for fractional differential problems, Math. Comput. Simul 147 (2017) 210-219.

[16] S. Esmaeili, M. Shamsi, A pseudo-spectral scheme for the approximate solution of a family of fractional differential equations, Commun. Nonlinear Sci. Numer. Simul. 16 (2011) 36463654.

[17] A. Pedas, E. Tamme, On the convergence of spline collocation methods for solving fractional differential equations, J. Comput. Appl. Math 235 (2011) 3502-3514.

[18] A. Saadatmandi, M. Dehghan, A Legendre collocation method for fractional integro-differential equations, J. Vibr. Contr. 17 (2011) 2050-2058.

[19] M. Delkhosh, Introduction of derivatives and integrals of fractional order and its applications, Appl. Math. Phys. 1 (2013) 103-119.

[20] M. Jleli, M. Kirane, B. Samet, A numerical approach based on In-shifted Legendre polynomials for solving a fractional model of pollution, Math. Methods Appl. Sci. 40 (2017) 7356-7367.

[21] A. Lotfi, S. A. Yousefi, A numerical technique for solving a class of fractional variational problems, J. Comput. Appl. Math. 237 (2013) 633-643.

[22] R. Caponetto, S. Fazzino, A semi-analytical method for the computation of the Lyapunov exponents of fractional-order systems, Commun. Nonlinear Sci. Numer. Simul. 18 (2013) 22-27.

[23] H. Khosravian-Arab, M. Dehghan, M. R. Eslahchi, Generalized Bessel functions: Theory and their applications, Math. Methods Appl. Sci. 40 (2017) 6389-6410.

[24] G. Wang, Twin iterative positive solutions of fractional qdifference Schrodinger equations, Appl. Math. Lett. 76 (2018) $103-109$.

[25] S. Ezz-Eldien, E. Doha, A. Bhrawy, A. El-Kalaawy, J. Machado, A new operational approach for solving fractional variational problems depending on indefinite integrals, Commun. Nonlinear Sci. Numer. Simul. 57 (2018) $246-263$.

[26] K. Sayevand, K. Pichaghchi, Efficient algorithms for analyzing the singularly perturbed boundary value problems of fractional order, Commun. Nonlinear Sci. Numer. Simul. 57 (2018) 136 168.

[27] K. Parand, M. Nikarya, J. A. Rad, F. Baharifard, A new reliable numerical algorithm based on the first kind of Bessel functions to solve Prandtl-Blasius laminar viscous flow over a semiinfinite flat plate, Zeit. Natur. A. 67 (2012) 665-673.

[28] K. Parand, S. Latifi, M. Delkhosh, M. Moayeri, Generalized Lagrangian Jacobi Gauss collocation method for solving unsteady isothermal gas through a micro-nano porous medium, Eur. Phys. J. Plus 133 (2018) 28.

[29] K. Parand, P. Mazaheri, M. Delkhosh, A. Ghaderi, New numerical solutions for solving Kidder equation by using the rational
Jacobi functions, SeMA J. 74 (2017) 569-583.

[30] S. Yuzbasi, M. Sezer, An improved Bessel collocation method with a residual error function to solve a class of Lane-Emden differential equations, Math. Comput. Model 57 (2013) 12981311.

[31] S. Yuzbasi, Numerical solution of the Bagley-Torvik equation by the Bessel collocation method, Math. Methods Appl. Sci. 36 (2013) 300-312.

[32] K. Parand, M. Nikarya, Application of Bessel functions for solving differential and integro-differential equations of the fractional order, Appl. Math. Model. 38 (2014) 4137-4147.

[33] Y. Xu, Z. He., The short memory principle for solving Abel differential equation of fractional order, Comput. Math. Appl. 64 (2011) 4796-4805.

[34] J. Gine, X. Santallusia, Abel differential equations admitting a certain first integral, J. Math. Anal. Appl. 370 (2010) 187-199.

[35] M. Markakis, Closed-form solutions of certain Abel equations of the first kind, Appl. Math. Lett. 22 (2009) $1401-1405$.

[36] T. Harko, M. K. Mak, Exact travelling wave solutions of nonlinear reaction-convection-diffusion equations, an Abel equation based approach, J. Math. Phys. 56 (11) (2015) 111501111525.

[37] F. Schwarz, Symmetry analysis of Abel's equation, Stud. Appl. Math. 100 (1998) 269-294.

[38] R. A. Adams, Sobolev Spaces, Academic Press, New York, 1975.

[39] W. W. Bell, Special Functions For Scientists And Engineers, Published simultaneously in Canada by D. Van Nostrand Company, (Canada), Ltd, 1967.

[40] B. Y. Guo, Spectral methods and their applications, World Scientific, Shelton Street, Covent Garden, London WC2H 9HE, 1998.

[41] J. Shen, T. Tang, L. L. Wang, Spectral Methods Algorithms, Analysics And Applicattions, first edition, Springer, 2001.

[42] K. Parand, M. Delkhosh, Accurate solution of the ThomasFermi equation using the fractional order of rational Chebyshev functions, J. Comput. Appl. Math. 317 (2017) 624- 642.

[43] K. Parand, M. Delkhosh, An efficient numerical solution of nonlinear Hunter-Saxton equation, Commun. Theor. Phys. 67 (2017) 483.

[44] J. P. Boyd, Chebyshev and Fourier spectral methods. 2nd ed, New York Dover, 2000.

[45] K. Parand, J. A. Rad, M. Nikarya, A new numerical algorithm based on the first kind of modified Bessel function to solve population growth in a closed system, Int. J. Comput. Math. 91 (2014) 1239-1254.

[46] K. Parand, M. Nikarya, A numerical method to solve the 1D and the $2 \mathrm{D}$ reaction diffusion equation based on Bessel functions and jacobian free Newton-Krylov subspace methods, Eur. Phys. J. Plus 132 (2017) 496-514.

[47] K. Parand, M. Nikarya, A novel method to solve nonlinear Klein-Gordon equation arising in quantum field theory based on Bessel functions and Jacobian free Newton-Krylov subspace methods, Commun. Theor. Phys. 69 (2018) 637.

[48] K. Parand, M. Delkhosh, M. Nikarya, Novel orthogonal functions for solving differential equations of arbitrary order, Tbilisi Mathematical Journal 10 (2017) $31-55$. 